\title{
An Efficient and Effective New Generation Objective Quality Model for Mobile Applications
}

\author{
Sobia Zahra \\ Department of Software Engineering, University of Engineering \& Technology, Taxila, Pakistan \\ Email: zahra.sobia@gmail.com \\ Asra Khalid \\ Department of Software Engineering, University of Engineering \& Technology, Taxila, Pakistan \\ Email: asra_05@yahoo.com \\ Ali Javed \\ Department of Software Engineering, University of Engineering \& Technology, Taxila, Pakistan \\ Email: ali.javed@uettaxila.edu.pk
}

\begin{abstract}
Recent proliferation of mobile market has swiftly increased the competition in mobile software market, new technology and new devices are emerging at phenomenal speed. As the number of mobile applications is increasing at daily rate, quality is becoming major issue. So mobile software organization need some quality model as guideline to improve and maintain quality of application under development. Mobile application mainly depends on user response and user acceptance so they need maintainability, usability, suitability etc. This research paper presents mobile application quality model focusing on key quality characteristics mainly extracted from ISO 9126 quality model, which effect quality of mobile applications. Furthermore some responsibilities of QA team in mobile application development are also discussed and lastly focused on the issue of 'tracking the quality of mobile applications after deployment'.
\end{abstract}

Index Terms-Mobile application, ISO 9126 Quality Model, Key Quality Characteristics, Mobile Application Development, Mobile Application Quality Model

\section{INTRODUCTION}

A study by Gartner Group [1] anticipate that projected no. of mobile apps to be downloaded in 2012 is 36 billion i.e. 5 app. per person over the globe. Another study [2] exemplifies that 428 million mobile devices were sold worldwide in 2011 Q1, a $19 \%$ increase from the previous year.

These statistical study shows that mobile phone is a vital necessity of our lives. Nowadays almost everyone owes his/her personal phone not only for messaging and calling purpose but also for entertainment, communication over the web, complex business transaction, instant accessibility of required information and much more.
Meadows [3] anticipate in his study that in mobile phones, smart phones are fastest emerging platform with anticipated 1.6 billion users by 2013 .

This rapid propagation has dramatically increased the need and growth of mobile app development. Resultantly for enterprises and individual developers this voracious appetite brings loads of opportunities [4]. But with the opportunities there comes challenges and it is not an easy task to get sensation success in today's flourishing field of mobile app development. In these challenges quality is the one of the biggest challenge where speed and performance of mobile apps are key factors for success of applications from users' perspective.

Developing mobile application is similar to desktop application but there are certain factors that make mobile applications different from desktop applications. The most critical difference between mobile application and desktop application is the way people use it. Mobile applications are usually small in size and are designed in a way that they use less power. So quality metrics for mobile applications also change due to these factors. Quality of mobile application is almost same as user experience of quality as life time of mobile applications are much less then desktop applications i.e. 3 to 4 weeks. So user will delete or change the application if it is not according to his needs and perception of quality [5]. There are many applications for same purpose in market and there is tough competition in market of mobile applications. Good quality of mobile is an attribute that promise a good response from customer.

In this research paper we highlighted the need of a quality model for mobile application development. We proposed a quality model as well as provided some guidelines for the Quality Assurance Team to follow for presenting a high quality mobile application to end- user and gain user satisfaction through quality product. In this research paper Literature review related to mobile applications is given in Section II. Quality model for mobile applications is proposed in Section III and key 
quality characteristics extracted from ISO9126 are defined in context of mobile applications. Only those characteristics are part of quality model which influence mobile application quality. Section IV presents some factors that QA team should take in account for better quality of mobile applications. While in Section $\mathrm{V}$ we have discussed some point about tracking the quality of mobile application after its deployment. All of these provide guideline for mobile App developer to produce high quality application and gain profit from them. Section VI concludes the research work.

\section{LITERATURE OVERVIEW}

Dominik Franke and Stefan Kowalewski [6] used software quality models of McCall's, Boehm and ISO9126 in their research work. They extracted some of the quality characteristics from these models and proposed a quality model for mobile applications. Afterwards they applied their quality model on two android applications to evaluate the quality of those mobile applications.

Aaron Hansen [7] reports tradeoff between speed and quality of mobile development and proposed a framework for quality acceleration. As Speed is the king in Mobile market and it is well understood that all software development processes are either time driven or quality driven. Due to lack of resources most of development processes are time driven. To ensure the quality of product, automated quality assurance process should be started at early phases of development, so that defects detected and fixed at start and there will be no cost of poor quality later on. Mobile devices have limited resources and more interoperability risks as well as speed is basic requirement. So Agile development is best for mobile apps development, QA process is involved at very start and bugs are removed resulting in high quality and high level of customer satisfaction and retention. In QA process automatic tools can be used to integrate QA with development process and reduce cost/time/risk of project. But automation is too expensive so outsourcing the product testing and utilizing a provider of effective QA process makes sense for competing in global market. This framework results in saving of cost and time, elimination of risk and uncertainty, doubling bug fix rate and most significant higher quality achievement.

John Garofalaki, Antonia Stefani, Vasilios Stefanis, Michalis Xenos [8] evaluated external quality attributes like functionality, usability, reliability and efficiency for M-Commerce system from user perspective and also answered the question "Can the M-commerce system be both well designed as well as high quality system?" Advantage of M-commerce system is that transaction is independent of location and time constraint. Three steps of user interaction with M-commerce system are presentation, navigation and purchasing. Presentation relates to how the product or service is presented to user, how text and images are organized. Navigation is accessing of M-commerce system information via indexes, tool bars, site maps and quick links. These component help system provide quality to end user, by means of easy-information access and easy search facility. Facilities provided for each commercial transaction comes under Purchasing. Quality in this step is achieved via authentication and personalization, also stability, alternative payment methods, error tolerance and error recovery count as quality factor. Evaluation of quality factors is done via heuristic evaluation of ISO9126 attributes. User interface is analyzed as good or bad. Mobile attribute weight (MAW) matrix is used in order to access a result from all the analyzed values. They get the result that the end users of $\mathrm{m}$ - commerce system give emphasis to usability and efficiency rather than reliability and functionality, M-commerce user always want to get efficiency in time and resource behavior.

Dominik Franke and Carsten Weise [9] contributed in mobile software quality and proposed a framework based on existing models, metrics, patterns, methods and tools for testing mobile applications. Generally used software design patterns can also be used in mobile apps. Like model view controller pattern helps in unit testing at view level and target-action pattern allows testing on plain and abstract level in mobile applications. Firstly a model should have a clear syntax and semantics to achieve flexibility, adaptability and portability. A finite state machine (FSM) is such a model with clear syntax and semantics. So developers can easily understand, learn and compare different platforms and test them. Initially quality of mobile application is analyzed statistically and empirically. Result of this analysis later used to define methods and tools for testing the quality lacks exposed in analysis phase. Then these tools and methods are applied in a case study to evaluate the framework and thus ensure conformance of quality in mobile application development.

Selim Ickin, Katarzyna Wac, and Markus Fiedler [10] reported that in any mobile application the thing that matter most is its user acceptance. User acceptance of a mobile application depends on the application's perceived experience, and the appropriateness of the application to the user's requirements. For this quantitative and qualitative procedures are used where the user becomes an active participant in the research. So to observe user experience of quality 30 android users are selected. For 30 days their usage of different mobile applications are observed. As result of this study some factors that affect any mobile application's quality are found which are: Application interface design, application performance, battery efficiency, phone features, application and connectivity cost, User routines and lifestyle and quality of service.

Josh Dehlinger and Jeremy Dixon [11] indicated that Mobile application development is much different from other application development due to some characteristics of mobile phones. The most important reasons of these differences are: mobile device user 
interface which is small and sometime touch sensitive, there are many mobile platforms (e.g. Android, Symbian etc.) and mobile computing is novel so not much work is done in mobile development process. Due to these reasons there are many challenges to mobile application engineering process which affect over all development of any mobile application. Different mobile phone has different sizes of screen so developing an application that can run on all mobiles is a challenge. There are many platforms of mobiles. Developing an application for all platforms is difficult. Most of mobile phones are context aware and movement sensitive, these features of mobile applications also affect application's requirement and development.in market there are many applications that perform same functionality so user change application if it is not according to his need. to compete others it's better that applications are self-adaptive which is also a challenge for developer.

Yousuf Hasan, Mustafa Zaidi, and Najmi Haider [12] added in research related to quality of mobile applications that Smart Phone market is unpredictable and there are many platforms for smart phones like android, iOS and windows phone. Developing applications for all the platforms are costly and time consuming infect impossible. One option is to use HTML5 for developing cross platform applications. Quality of HTML5 based application development is measured in their work. To measure quality ISO 9126 standard is used. ISO 9126 standard has six major factors, which are Efficiency, Maintainability, Reliability, Functionality, Usability, and Portability. HTML5 based applications development cost is also measured in this paper. For this purpose a project which was being developed in HTML5 is used. Time required for developing the project is measured and it is compared with other project's development time. It was found that HTML5 based application is $67 \%$ cost effect. According to this research result HTML5 can be used for developing cross platform applications for smart phones without compromising on quality or cost.

In paper of Anthony I. Wasserman, Carnegie Mellon Silicon Valley [13] they discussed software engineering issues related to mobile devices application development. Traditional application development processes cannot be used while developing mobile applications because these applications are different from traditional applications. A proper method of developing mobile application should be used and these applications should be tested after development. Most mobile applications are small. Mobile applications can be divided into two categories one of those are applications that runs only on mobile and require no internet and other one are those that are web based and require internet. There are some tools for different platform's application development but these tools can be used only to develop small applications for large applications we have to follow proper software engineering process.

Due to small screen size, need of interaction with other applications in mobile (e.g. incoming call while playing a game), sensor handling (movement sensitiveness), different platforms for different mobiles, battery, and security mobile applications are different from desktop applications [13].

Mobile device applications [14] are generally used frequently but for small interval. Due to these characteristics good mobile phone applications provide dedicated and efficient capability for accomplishing particular functionality that is for general environment. Some important characteristic of good mobile applications are responsive to user input, fast startup time, and defined purpose. These characteristics are important for a highly productive user experience while using mobile applications. Developer has to take care of these aims when planning, developing, and testing mobile application. Mobile devices are different from desktop and laptop computers with respect to their architecture because mobile phones don't have any hard disk like computers so they work only with their RAM. They use RAM for storage and also for execution of programs and applications. There is flash memory in mobiles for long duration storage purpose. There is no concept of paging in mobile devices so while developing mobile applications memory management is very important as RAM and flash storage is limited in mobile applications. Mobile applications use internet more frequently then desktop applications so mobile applications should be more reliable. It should manage resources and avoid memory leakage to increase its performance.

Good application should be useable [15] any time at any place with up to date information and usefulness. There should be time aware options and relevant content round the clock. While developing mobile applications personalization should also be considered. User wants from mobile applications that they behave how they want them to behave. User can change mobile application according to his requirement [16] i.e. how buttons are placed on screen and how to navigate between screens,

\section{MOBILE APPLICATION QUALITY MODEL}

Mobile application development is quite similar to development of other applications but there are some variations, in both developments, which count a lot in quality of mobile applications. Some of these distinctions of mobile environment are user interface, performance, security, battery power, hardware integration, multiple plate form etc. Organizations involve in mobile application development want to improve user experience but diversity of operating system, devices and networks are major bottlenecks in their way. So these organizations need to follow a quality standard for mobile applications, not only to validate functionalities but also to provide user with high quality mobile applications.

Traditional ISO9126 quality model [17] covers all the quality attributes for desktop and embedded applications, 
but these attributes are numerous and not all to be applied on mobile application development. In this research paper we will provide a quality model defining key quality characteristic related to mobile applications. These quality characteristics are actually extracted from ISO9126 quality model and these characteristics specifically affect quality of mobile applications. If a developer keeps these things in mind while developing mobile applications, he will end up with better quality software.

Some quality characteristics of mobile applications are device specific while other characteristics are quite general in nature. Device dependent characteristics of mobile applications are screen size, memory, processing power, battery etc. Other quality characteristics that effect mobile application's quality are portability, extensibility, adaptability, efficiency, maintainability, usability, data integrity etc.

\section{A. Maintainability}

There are a lot of mobile companies and each company is providing different mobiles with different platforms and different features. Mobile application should be flexible to run on different hardware and different configuration settings as well as to install updates available in App Markets as new versions of existing platform come in market frequently. That's why mobile development platforms [6] provide relative layout instead of absolute one. So that applications can work with different platforms in different mobiles.

\section{B. Extensibility:}

Extensibility [6] can be functional as well as nonfunctional. Functional extension is to add new function and non-functional extension to add some usability factor but not functionality. If underlying platform of mobile extends then mobile application is also extended to add updated functionality or it can be extended if updates available in App Market.

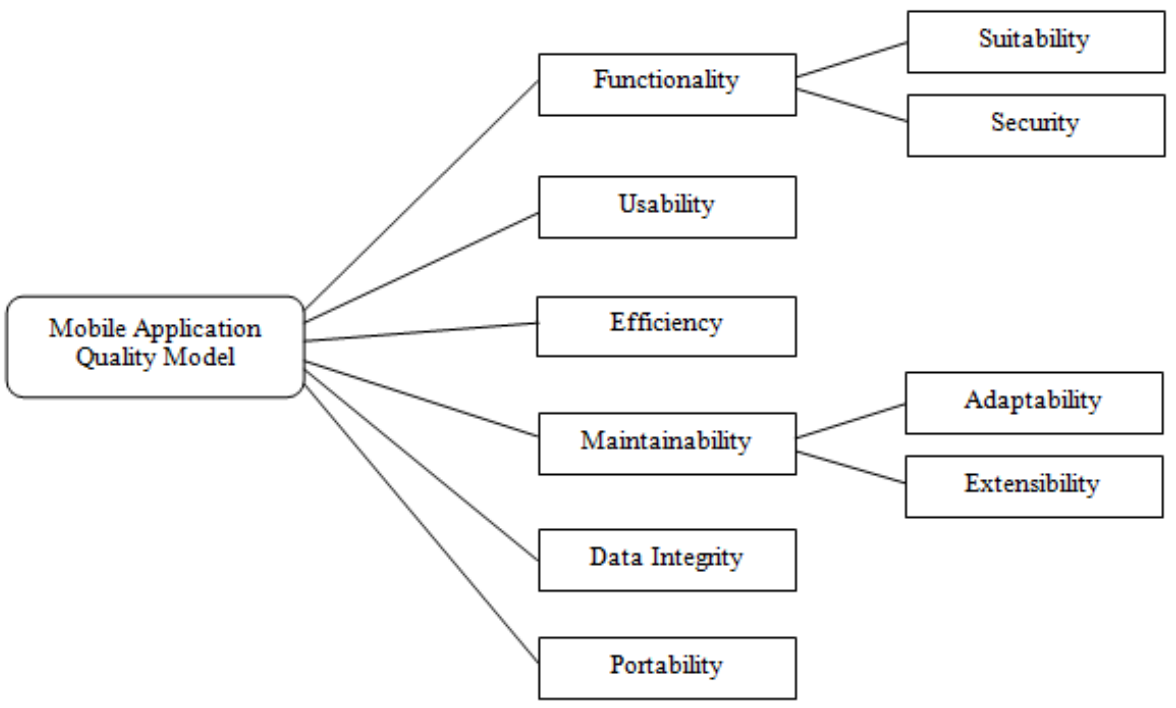

Figure 1. Mobile applications quality model

\section{Adaptability [17]}

Adaptability is extent to which application adapts changes from its environment like adaptability in input methods and different orientations of handheld devices (horizontal and vertical view). It also include remembering general words mostly used by user while using application i.e. user name.

\section{Usability}

It is ease with which [17] user can learn and understand mobile application and user-friendliness of application. Usability is all about improving user experience and response about particular application. First 30-60 seconds of mobile application are crucial as user decides either to continue with the application or quit the application and move forward to some other application in App market.
If a mobile application provide difficult interface mobile user will switch to 'an easy to use' application within no time. It is how easy is to use application i.e. easiness in giving input and getting an understandable output.

Mobile screen is very small in size and a lot of sizes are available in market and new dimensions for screen sizes come every day in market. In mobile application user cannot open two screens at same time he has to work on single application at a time and have to perform continuous switching to use many applications at a time. So developer should design an application which is easy to use in such limited screen size.

When we talk about input capabilities of mobile it is much limited then standard QWERTY keyboard. User use mobile while moving and give input with much less concentration so way of getting input should be such that there are less chances of errors. For example keyboard should convert in numeric form when some 
number is required as input. And developer should try to add least requirements for special character input because it is complex to enter symbol in mobile keyboard this thing make application less user friendly.

Application should follow standard convention for general options like close button is always on right side in mobile applications.

\section{E. Portability[17]}

Portability is important factor in mobile apps which measures if an application have tendency to run on multiple platforms or not. This can be measured by running the application on multiple devices and multiple platforms. Mobile market is expanding day by day and there are numerous models for each handheld device, so user should be capable of running the same application on different mobile phone with same efficiency, when he/she switches from one device or platform to other.

\section{F. Efficiency[17]}

Efficiency relates to the response time of the application as well as optimal use of mobile resources. Resources might include other software, hardware, disks etc. So in case of mobile, efficiency of an application is dependent upon battery power and memory of mobile phone.

\section{G. Data Integrity [6]}

Data Integrity is the ability for a mobile to keep information even after mobile application is paused or killed, this feature is essential for mobile applications as when a call arrives, current state and information of running application should be saved.

\section{H. Functionality}

It is extent to which software fulfills user requirement and specification documented in requirement specification phase. In mobile application functionality is to provide an application according to end user requirement in custom application software. While in generic applications, development focus is on the general needs of end users in particular area.

\section{Suitability}

One of the quality factors of functionality is Suitability which checks the extent to which an application fulfills needs of a particular user without devastating the user. Therefore mobile applications should attempt to minimize the duration needed to complete an activity by user.

\section{J. Security}

Security is very vast concept in mobile applications. When user uses any mobile application all the user's data operated by application should be secure. When application run on operating system it may use some features of operating system, application should be able to run without effecting operating system. Application should also be able to keep its feature and data secure from operating system as well.

Most of mobile applications use internet while running developer should take care of security of data while interacting through internet.

It is up to developer to choose desired key quality attribute or modify this model according to his priorities.

\section{IMPORTANT ROLE OF QA TEAM IN MOBILE APPLICATION QUALITY}

An organization can end up with high quality software if quality assurance (QA) of mobile application is conducted on regular interval. QA team should perform quality assurance activities throughout development of mobile App. But the most important factor that QA team should consider, and that put value to quality of mobile application are as follows:

\section{A. Validate End user Requirement}

If the application provides all the non-functional requirements like portability, security, efficiency but is unable to fulfill the basic need of user, then the application is simply useless. So, before deployment of an application into App market, major responsibility of QA team is validate if the mobile application is fulfilling end user's requirements or not. For that QA team should take into account their testing methods for functional validation of applications, but these applications run on multiple platforms and multiple devices, so manual testing is not efficient. Consequently QA team should use some automated tool for validation of functional requirements of an application.

\section{B. Monitor Mobile application production:}

As QA is an umbrella activity so it is better for QA team to test end to end service health and functionality of mobile application throughout application's development phase. This approach is better to avoid major defects and end user problems by early identification and removal of these defects. This will help organization to provide user with high quality applications according to user requirements.

\section{Validate performance of Mobile application}

Performance in mobile applications is more crucial than any other desktop or embedded application as user expects fast and efficient processing on handheld devices. In handheld devices single early failure in an application counts for non-returning user and there is no big loss to an organization besides bad perception of their application in the market. So QA team needs to validate if the application performs well in all the situations, normal as well as critical situation. 


\section{D. tracking quality of mobile application after deployment}

In smart phone App market, developer can keep track of his/her mobile App. quality and he can improve the quality by considering few factors.

1. Make a good publicity campaign of an application and increase its visibility, which ultimately result in higher likelihood, better rating and increased download rate all for a better quality mobile application.

2. Keep into consideration user's response and listen to their feedback by reading forum and comments about your application. User's response can also be evaluated by tracking daily download rate and potential active installs.

3. Provide user with bug/crash reporting mechanism by which they can report any defect/error found in the application. And ultimately developer will be able to get direct feedback of his/her application.

4. Check for the usability of an application by performing usability/hallway testing of the application and check user response. Find out the areas where user get stuck or get confused about how to proceed further. Also keep an eye on user response and emotional behavior when they are using the application.

\section{CONCLUSIONS}

This research paper proposes a quality model for mobile application development. This quality model is actually derived from ISO 9126 quality model and it can help mobile development organizations to develop high quality mobile applications. This is general model for mobile applications; developer can modify this quality model according to specific application they are working upon. This paper also point out some factors that QA team should check during development of mobile application to provide end user with a better quality mobile apps. Some points, to track quality of mobile application after deployment, are also discussed in this research paper.

\section{REFERENCES}

[1] "Gartner Says More than 1 Billion PCs In Use Worldwide and Headed to 2 Billion Units by 2014" Gartner Website. [Online]. http://www.gartner.com/it/page.jsp?id=703807

[2] "Gartner Says 428 Million Mobile Communication Devices Sold Worldwide in First Quarter 2011, a 19 Percent Increase Year-on-Year" Gartner website. [Online]. http://www.gartner.com/it/page.jsp?id=1689814

[3] "Evolution of the Smartphone"Larry Meadows. HTC Website. [Online]. http://blog.htc.com/2012/10/evolution-of-thesmartphone/
[4] "Mobile Applications - Quality Matters" Dave Donovan. EzineArticles. [Online]. http://ezinearticles.com/?Mobile-Applications--Quality-Matters\&id=6666537

[5] Katarzyna Wac, Markus Fiedle Selim Ickin, "Factors Influencing Quality Of Experience Of Commonly Used Mobile Applications," IEEE Communications Magazine , April 2010. DOI:10.1109/MCOM.2012.6178833

[6] Dominik Franke and Stefan Kowalewski, "A Mobile Software Quality Model," in 12th International Conference, 2010. DOI:10.1109/QSIC.2012.49

[7] Aaron Hansen, "A Mobile Software Quality Framework," Lionbridge Technologies, 2007.

[8] Antonia Stefani, Vasilios Stefanis, Michalis Xenos John Garofalaki, "Quality Attributes Of ConsumerBased M-Commerce Systems," in IEEE International Conference, 2007.

[9] Dominik Franke and Carsten Weise, "Providing A Software Quality Framework For Testing Of Mobile Applications," in Fourth IEEE International Conference, 2011. DOI:10.1109/ICST.2011.18

[10] Katarzyna Wac, and Markus Fiedler Selim Ickin, "Factors Influencing Quality of Experience of Commonly Used Mobile Applications," IEEE Communications Magazine, April 2012. DOI:10.1109/MCOM.2012.6178833

[11]Josh Dehlinger and Jeremy Dixon, "Mobile Application Software Engineering: Challenges and Research Directions".

[12] Mustafa Zaidi, Najmi Haider Yousuf Hasan, "Smart Phones Application development using HTML5 and related technologies: A tradeoff between cost and quality," International Journal of Computer Science Issues, 2012.

[13] Carnegie Mellon Silicon Valley Anthony I. Wasserman, "Software Engineering Issues for Mobile Application Development," FoSER, November 2010.

[14]Characteristics of Mobile Applications, http://ptgmedia.pearsoncmg.com/images/03212693 14/samplechapter/salmre_ch02.pdf

[15] "Some Essential Characteristics of Mobile Applications"

http://ittechsols.wordpress.com/2012/12/18/someessential-characteristics-of-mobile-applications/

[16] "6 Major Characteristics of Mobile Apps" http://www.flamelab.de/article/6-majorcharacteristics-of-mobile-apps/

[17] Software engineering - Product Quality - Part1: Quality Model, International Standard Organization Std. 9126, 2001

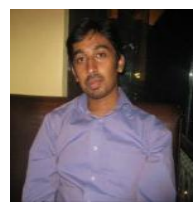

Engr. Asra Khalid is a MS Scholar as well as Research Associate in the Department of Software Engineering at University of Engineering and Technology Taxila, Pakistan. She 
graduated from University of Engineering and Technology Taxila in Software Engineering in July 2012. Her areas of interest are Mobile Application Development, Computer Graphics, Desktop Application Development, Object Oriented programming, Software Design \& Architecture.

Engr. Sobia Zahra has completed her BS Software Engineering degree in July 2012 from University of Engineering and Technology Taxila, Pakistan. Her areas of interest are Mobile Application Development, Web Development, Databases, Software Quality Assurance, Software Requirement Engineering and Operating System. Currently she is MS Scholar as well as Research Associate in the Department of Software Engineering at University of Engineering and Technology, Taxila, Pakistan.

Engr. Ali Javed is serving as an Assistant Professor in the Department of Software Engineering at UET Taxila, Pakistan. He is also a $\mathrm{PhD}$ Scholar in Computer Engineering Department at UET Taxila, Pakistan. He has received his MS degree in Computer Engineering from UET Taxila, Pakistan in February, 2010. He received Chancellor's Gold Medal in MS Computer Engineering degree and became the first MS student in the history of UET Taxila to be awarded Chancellor's Gold Medal. He has received B.Sc. degree in Software Engineering from UET Taxila, Pakistan, in September, 2007. He got 3rd position in Software Batch-2003F in BS Software Engineering degree. His areas of interest are Digital Image Processing, Computer vision, Video Summarization, Mobile Application Development, Software Requirements Engineering, Software Quality Assurance and Software testing. 